\title{
MBL2 gene polymorphisms increase the risk of adverse neurological outcome in preterm infants: a preliminary prospective study
}

\author{
Cinzia Auriti', Giusi Prencipe ${ }^{2}$, Barbara Caravale ${ }^{3}$, Maria Franca Coletti ${ }^{4}$, Maria Paola Ronchetti', Fiammetta Piersigilli', \\ Chiara Azzari ${ }^{5}$ and Vincenzo M. Di Ciommo
}

BACKGROUND: As described in animal models, the lectincomplement pathway is central to the propagation of ischemia-reperfusion injuries in many tissues, including the brain. Similarly, it might affect the genesis of brain damage in preterm infants. MBL2 gene single-nucleotide polymorphisms (SNPs), regulating mannose-binding lectin (MBL) serum levels, could predict the risk of adverse neurological outcome in these infants.

METHODS: To evaluate the association between SNPs of the MBL2 gene and long-term neurological outcomes in preterm infants, 75 infants (gestational age $(G A) \leq 32 \mathrm{wk}$ ) were observed in a prospective longitudinal study and assessed by clinical and instrumental exams at 12 and 24 mo of corrected age (CA). They were genotyped for the promoter polymorphism -221 and for the exon- 1 variant alleles (at codons 52, 54, and 57) of the MBL2 gene.

RESULTS: The MBL2 exon- 100 genotype was more frequent in children with an adverse neurological outcome $(5 / 35 ; 7 \%)$ than in controls $(0 / 40 ; 0 \%), P=0.045$. The risk of intraventricular hemorrhage in carriers of the genotype $\bigcirc O$ was marked, without reaching statistical significance (odds ratio: 8.67; 95\% confidence interval: 0.87-86.06; $P=0.07$ ).

CONCLUSION: Preterm infants who are carriers of MBL2 exon-1 $\bigcirc \bigcirc$ genotype are exposed to an increased risk of adverse neurological outcomes.

$\mathrm{T}$ he improved survival of preterm infants, and in particular of the extremely preterm infants (gestational age (GA) < $28 \mathrm{wk}$ ) (1), in recent years has increased their risks of adverse neurological outcomes, due to prenatal, perinatal, and postnatal determinants (2). They act through multiple, complex gene-environmental interactions, with hypoxia/ischemia and inflammation/infection mechanisms apparently playing a major role (3). In this context, certain proteins of innate immunity and variations of genes, regulating their production, could influence the chain of events that impact on neurological outcomes of preterm infants. Mannose-binding lectin (MBL) is a serum recognition molecule in the collectin family of the innate immune system. MBL serum levels are genetically determined, as some authors have described (4-6), and Sorensen underlined in an elegant study on adult twins, in which he estimated the heritability of serum MBL levels (5). Three structural single-nucleotide polymorphisms (SNPs) of the $M B L 2$ gene exon- 1 , at codons 52,54 , and 57 , interfere with the assembly of the protein, causing decreased functional circulating MBL $(4,5)$. In addition, the $-221 \mathrm{X} / \mathrm{Y}$ promoter SNP affects the protein expression, with the $-221 \mathrm{Y}$ variant being associated with high MBL levels in the serum (6).

The lectin pathway of the complement cascade begins with MBL-mediated recognition of terminal oligosaccharides presented on the surface of a target cell, micro-organisms or cells belonging to an injured tissue. By binding to sugar moieties of the surface of the cell, MBL activates the lectin-complement pathway (7). In the case of micro-organisms, MBL promotes the agglutination and clearance of pathogens by phagocytes, providing, in a short time, the protection of the host against the invasion. At birth, this innate mechanism of immunity plays a critical role in the defense against infections (8). It quickly responds to early, postnatal bacterial invasion, combating many species of pathogens. In the case of tissue damage, MBL rapidly deposits on target cells and triggers downstream complement activation in the acute phase, enhancing the cleavage of C3 (9). In patients in the acute phase of the stroke, MBL seems to contribute to inflammatory cerebral injury as well as to play a direct role in stroke recovery $(10,11)$. Furthermore, several studies in animal models have shown that MBL-mRNA is expressed in brain tissue (12) and the lectin pathway is central to the propagation of ischemia-reperfusion injury across a variety of tissues $(13,14)$.

In humans, at birth, circulating MBL is lower in preterm than in more mature infants, being influenced by GA, in addition to

'Department of Neonatology, Bambino Gesù Children's Hospital (IRCCS), Rome, Italy; ${ }^{2}$ Rheumatologic Research Laboratory, Bambino Gesù Children's Hospital (IRCCS), Rome, Italy; ${ }^{3}$ Department of Developmental and Social Psycology, La Sapienza University, Rome, Italy; ${ }^{4}$ Department of Developmental Psychology, Bambino Gesù Children's Hospital (IRCCS), Rome, Italy; ${ }^{5}$ Department of Pediatrics, Institute Anna Mayer, Florence, Italy; ${ }^{6}$ Epidemiology Unit, Bambino Gesù Children's Hospital (IRCCS), Rome, Italy. Correspondence: Cinzia Auriti (cinzia.auriti@opbg.net) 
the genotype (15-18). Its levels progressively increase, in the first weeks-months of postnatal age.

The aim of this study was to explore the association between SNPs of the MBL2 gene and neurodevelopmental outcomes, diagnosed by motor, sensorial, and cognitive assessments, in a group of preterm infants, observed prospectively, until 24 mo of corrected age (CA).

\section{RESULTS}

Seventy-five premature infants, 39 (52.0\%) males, completed the follow-up program up to 24 mo of CA. Their mean birth weight was $1,202 \mathrm{~g}( \pm 404)$. Among these neonates, 29 (38.7\%) had a GA $<28 \mathrm{wk}, 36(48,0 \%)$ between 28 and $31 \mathrm{wk}$ and 10 (13.3\%) were $32 \mathrm{wk}$ infants. The mean GA of all infants observed in the study was $28.6 \mathrm{wk}( \pm 2.3)$. Forty-three $(57.3 \%)$ neonates developed at least an episode of nosocomial sepsis (NS) during the hospital stay, 14 (18.7\%) were affected by necrotizing enterocolitis at the time of admission in the neonatal intensive care unit or developed this disease during hospitalization, 19 (25.3\%) showed intraventricular hemorrhage (IVH), grades II to IV and 23 (30.7\%) showed periventricular leukomalacia (PVL) (12 neonates, 16.2\%, showed both) at ultrasound (US) examination during the hospitalization or subsequently. At 24 mo of life, 16 (21.3\%) children had abnormal visual function, $10(13.3 \%)$ showed from mild to severe abnormal auditory function, 35 (46.7\%) showed from mild to severe neurological disorder and/or mental developmental index (MDI)/psychomotor developmental index (PDI) $\leq 70$ at Bayley's II scales.

According to the definition of adverse neurological outcome, 35 of 75 (46.7\%) had an adverse neurological outcome.
Among them, 8 of 35 (22.8\%) children had a single adverse outcome, 4 abnormal clinical neurological examination, and 4 MDI and or PDI $\leq 70$ at Bayley's II scales. Twenty-seven of 35 (77.2\%) children had more than an outcome: serious abnormal neurological examination or low Bayley score, or both of them, with or without visual or auditory major deficits. The other 40 children had a normal development, 5 of them with minimal visual or hearing impairment.

Table 1 shows characteristics of children who completed the follow-up program, by adverse neurological outcome. Risk factors strongly associated with the main outcome were, as expected, PVL, IVH, antiepileptic therapy, and visual impairment. The genotypes distribution in our neonates was similar to that in general Caucasian population: AA 50/75 (66.7\%); AO 20/75 (26.7\%), and OO 5/75 (6.7\%) (19). We have genotyped four common SNPs of the MBL2 gene: codons 52, 54, and 57 in the exon-1 and -221 in the promoter region. Allele and genotype frequencies for all of the studied SNPs are summarized in Table 2. The allelic frequencies of the promoter -221 polymorphism $\mathrm{X}$ and of the exon 1 polymorphisms $\mathrm{C}$ and $\mathrm{B}$ were similar in children with adverse neurological outcome and in normal children. Naming "O" the MBL-2 structural variant alleles $\mathrm{B}$ and $\mathrm{C}$, the $M B L 2$ exon-1 OO genotype associated with MBL deficiency was more frequent in children with an adverse neurological outcome $(5 / 35 ; 7 \%)$ than in normal children $(0 / 40 ; 0 \%), P=0.045$ (Table 2). The result was similar in the group of 65 extremely $(<28 \mathrm{wk})$ and very preterm $(<32 \mathrm{wk})$ infants (5/32; 15.6 vs. $0 / 33 ; 0 \%$ respectively, $P=0.056)$. In fact, all these five infants with OO genotype were extremely (4/5) or very preterm babies (1/5) and have been affected by at least

Table 1. Clinical characteristics and procedures in 75 neonates enrolled in the study, by adverse neurological outcome

\begin{tabular}{|c|c|c|c|}
\hline Clinical characteristics & $\begin{array}{c}\text { No adverse outcome, } \\
\quad n=40(53.3 \%)\end{array}$ & $\begin{array}{c}\text { Adverse neurological } \\
\text { outcome, } n=35 \text { (46.7\%) }\end{array}$ & $P$ \\
\hline Gender (males) ${ }^{\mathrm{a}}$ & $20(50.0)$ & $19(54.2)$ & 0.71 \\
\hline Gestational age $<28 w^{a}$ & $13(32.5)$ & $16(45.7)$ & 0.36 \\
\hline Gestational age $=28-31 \mathrm{wk}$ & $20(50.0)$ & $16(45.7)$ & \\
\hline Gestational age $=32 \mathrm{wk}$ & $7(17.5)$ & $3(8.6)$ & \\
\hline Gestational age (weeks) ${ }^{\mathrm{b}}$ & $28.8 \pm 2.3$ & $28.1 \pm 2.4$ & 0.19 \\
\hline Birth weight $(g)^{b}$ & $1224 \pm 384$ & $1439 \pm 416$ & 0.39 \\
\hline Delivered by Cesarean section ${ }^{\mathrm{a}}$ & $29(72.5)$ & $19(54.3)$ & 0.15 \\
\hline Apgar $5 \min ^{b}$ & $7.8 \pm 1.4$ & $7.3 \pm 1.3$ & 0.15 \\
\hline $\mathrm{nCPAP}+\mathrm{MV}\left(\right.$ days) ${ }^{\mathrm{b}}$ & $21.6 \pm 19.8$ & $28.8 \pm 26.1$ & 0.23 \\
\hline Nosocomial sepsis ${ }^{a}$ & $19(47.5)$ & $24(68.6)$ & 0.08 \\
\hline Necrotizing enterocolitis ${ }^{a}$ & $5(12.5)$ & $9(25.7)$ & 0.22 \\
\hline Length of stay (days) ${ }^{b}$ & $53.43 \pm 37.1$ & $62.85 \pm 40.1$ & 0.48 \\
\hline Intraventricular hemorrhage ( $2-4$ grade $)^{\mathrm{a}}$ & $3(7.5)$ & $16(45.7)$ & $<0.001$ \\
\hline Periventricular leukomalacia ${ }^{a}$ & $8(20.0)$ & $15(42.9)$ & 0.03 \\
\hline Epilepsy therapy ${ }^{a}$ & $1(2.5)$ & $7(20.0)$ & 0.02 \\
\hline Visual impairment ${ }^{a}$ & $2(5.0)$ & $14(40.0)$ & $<0.01$ \\
\hline Hearing loss ${ }^{a}$ & $3(7.5)$ & $7(20.0)$ & 0.17 \\
\hline
\end{tabular}

${ }^{a} \chi^{2}$ test or Fisher test, as appropriate. ${ }^{b}$ Continuous variables are reported as mean $\pm S D ; P$ value has been calculated by $t$-test or Mann-Whitney test, as appropriate. $\mathrm{MV}$, mechanical ventilation with orotracheal tube; nCPAP, nasal continuous positive airway pressure. 
Table 2. Distribution of allele and genotype frequencies of three of four single-nucleotide polymorphisms in the MBL2 gene among neonates with and without adverse neurological outcome

\begin{tabular}{|c|c|c|c|}
\hline Alleles & $\begin{array}{c}\text { No adverse } \\
\text { neurological } \\
\text { outcome, } n=40\end{array}$ & $\begin{array}{c}\text { Adverse } \\
\text { neurological } \\
\text { outcome, } n=35\end{array}$ & $P^{a}$ \\
\hline Promoter alleles & & & 0.281 \\
\hline \multicolumn{4}{|l|}{$\begin{array}{l}-221 \mathrm{G} \rightarrow \mathrm{C} \\
\text { (allele X) }\end{array}$} \\
\hline G & $62(77.5)$ & $60(85.7)$ & \\
\hline C & $18(22.5)$ & $10(14.3)$ & \\
\hline \multicolumn{4}{|l|}{$\begin{array}{l}\text { Structural exon-1 } \\
\text { alleles }\end{array}$} \\
\hline $\begin{array}{l}\text {-Codon } 54 \\
\mathrm{G} \rightarrow \mathrm{A} \text { (allele B) }\end{array}$ & & & 0.217 \\
\hline G & $69(86.25)$ & 54 (77.14) & \\
\hline A & $11(13.75)$ & $16(22.86)$ & \\
\hline $\begin{array}{l}\text {-Codon } 57 \\
\mathrm{G} \rightarrow \mathrm{A} \text { (allele } \mathrm{C} \text { ) }\end{array}$ & & & 0.907 \\
\hline G & 79 (98.75) & $68(97.14)$ & \\
\hline A & $1(1.25)$ & $2(2.86)$ & \\
\hline \multicolumn{4}{|l|}{$\begin{array}{l}\text {-Codon } 52 \\
\mathrm{C} \rightarrow \mathrm{T} \text { (allele D) }\end{array}$} \\
\hline C & 0 & 0 & \\
\hline $\mathrm{T}$ & 0 & 0 & \\
\hline \multicolumn{4}{|l|}{ Genotypes } \\
\hline \multicolumn{4}{|l|}{$\begin{array}{l}\text { Promoter - } 221 \\
\text { genotypes }\end{array}$} \\
\hline YY & $22(55.0)$ & $25(71.4)$ & 0.219 \\
\hline$X Y$ & $18(45.0)$ & $10(28.6)$ & \\
\hline \multicolumn{4}{|l|}{$\begin{array}{l}\text { Structural } \\
\text { exon-1 } \\
\text { genotypes }\end{array}$} \\
\hline AA & $28(70.0)$ & $22(62.86)$ & 0.045 \\
\hline $\mathrm{AO}$ & $12(30.0)$ & $8(22.86)$ & \\
\hline $\mathrm{OO}$ & $0(0)$ & $5(14.28)$ & \\
\hline
\end{tabular}

Data are expressed as number and (\%). ${ }^{a} \chi^{2}$ test.

an episode of NS. Analyzing data by logistic regression model, IVH was associated with the adverse neurological outcome (OR: 10.4; 95\% CI: 2.7-40.1; $P=0.001$ ), even after adjusting for genotype, as expected, being in its deterministic pathway. With IVH (2-4 grade) as dependent variable, adjusting for NS as potential confounder, the risk of the genotype OO was marked, although not reaching statistical significance, while infection was a definite independent risk factor (OR: $4.31 ; P=$ 0.04) after adjustment (Table 3).

\section{DISCUSSION}

In a group of preterm infants with a GA $\leq 32 \mathrm{wk}$, we observed that homozygosity for SNPs in the exon 1 of the MBL2 gene was associated with an adverse neurological outcome at 12 and/or 24 mo of CA and may be predictive of neurological
Table 3. Multivariate analysis: genotypes of $M B L 2$ exon- 1 as a risk factor of IVH (grades 2-4 according to Papile) adjusted for confirmed NS

\begin{tabular}{lcc}
\hline & \multicolumn{2}{c}{ IVH } \\
\cline { 2 - 3 } Risk factors & OR $(95 \% \mathrm{Cl})$ & $P$ \\
\hline MBL2 genotype OO vs. AA+AO & $8.67(0.87-86.06)$ & 0.07 \\
Confirmed NS & $4.31(1.09-17.01)$ & 0.04 \\
\hline Cl, confidence interval; IVH, intraventricular hemorrhage; NS, nosocomial sepsis; OR, \\
odds ratio.
\end{tabular}

risks. To our knowledge, this is the first study in which this association has been explored among preterm babies. Our data add to the knowledge about genetic predisposition to more severe neurological outcomes of prematurity, which may be a marker of individual weakness. To evaluate the strength of clinical factors and/or genetics in predisposing preterm infants to develop neurological damage is a challenge. Many preterm infants have less favorable neurodevelopmental outcomes than others, with the same clinical risk factors. The complement system is an integral component of the innate immune system, that has been implicated in the physiopathology of the susceptibility to traumatic brain injuries (TBI) and acute stroke in animal models and in humans (20). Yager et al. (20) studying an experimental model of TBI in mice, found that MBL deficiency exacerbates acute CA3 (Cornu Ammonis) cells death and cognitive dysfunction, independently from complement activation. They demonstrated also that MBL contributes to brain size in adult mice, suggesting an endogenous neuroprotective role for MBL, and a heretofore unknown functional linkage between innate immunity and neurological outcome after TBI. In addition, Cevrera et al. (12) genotyped 135 adult patients (mean age $>70 \mathrm{y}$ ), with a brain stroke. At 3 mo of follow up, they concluded that genetically defined MBL deficiency was associated with a better outcome after acute stroke, without finding a matched increased risk of infections in MBL-deficient patients. Moreover, patients with MBL low genotypes disclosed lower serum levels of complement component 3 (C3) and complement component 4 (C4) than patients with MBLsufficient genotypes. Ducruet et al. (21) in a murine model of middle cerebral artery occlusion, have recently demonstrated the neuroprotective effect for genetic MBL mutation in the acute poststroke, but without improvements in either infarct volume and neurological function at $7 \mathrm{~d}$ examination. Finally, Heyer et al. (22) in a recent study, explored the relationship between the G/C SNP of the MBL2 gene and cognitive disfunction (CD) after carotid endoarterectomy (CEA) in 252 adult patients. The replacement of $\mathrm{G}$ by $\mathrm{C}$ in the promoter region of the MBL2 gene on chromosome 10 leads to an $\mathrm{X}$ to $\mathrm{Y}$ amino acid change resulting in a low expression of $M B L 2$ and reduced serum concentration in the range of a $25-50 \%$ decrease per deleterious allele. Although the univariate analysis suggested that the G/G MBL2 genotype might correlate with higher incidence of $\mathrm{CD}$ compared to the $\mathrm{C} / \mathrm{G}$ and $\mathrm{C} / \mathrm{C}$ genotypes, the final logistic regression model did not confirm such association at 1 mo after CEA. 


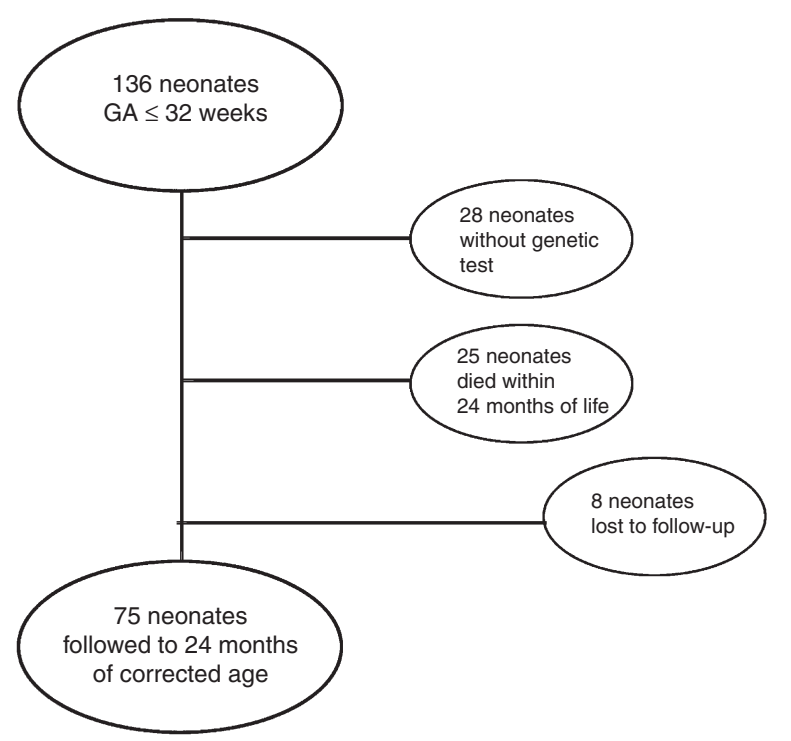

Figure 1. The study flow chart. GA, gestational age.

These results are in conflict with each other. Whether MBL has a protective or harmful role in the physiopathology of the ischemia reperfusion brain damage is still unclear.

In our study, all five preterm infants, carriers of the $\mathrm{OO}$ MBL2 exon-1 genotype, were extremely preterm $(n=4)$ and preterm $(n=1)$ infants. This could be a coincidence, given the low number of patients observed. Nonetheless Annells (23) suggested a possible causal relationship between a genetic variant in the human gene MBL2 and the risk of preterm birth.

Bodamer subsequently supported this hypothesis showing that neonate carriers of genotypes most likely conferring deficient MBL levels (OO) were significantly higher in the preterm birth group (24). We can speculate that fetal MBL2 genotype might be an additional genetic factor contributing to the risk of premature delivery, as fetal MBL produced by the fetal liver might play a role in the defense of pathogens and/or modulation of immune response during gestation.

All our babies with the OO genotype had at least an infection during the hospitalization, and finally an adverse neurological outcome. In previous studies, we have shown that preterm infants genetically deficient in MBL, but also those with genotypes wild type with low serum levels due to prematurity, have an high risk of infection, which in itself has a detrimental effects on mental and motor development in children born very prematurely (25). Therefore, the effect of deficiency of MBL on neurological development could be indirect in these infants. Indeed, brain damage induced by MBL deficiency may be partially independent from complement cascade in preterm infants, less active than in more mature babies and in adults. Other MBL-mediated mechanisms, closely related to the condition of severe prematurity, in particular to the marked brain immaturity of neonates, may have a role in the genesis of the neurological damage. Studies on the rat cerebellum performed by Zanetta and other authors (26-28) suggested a role for MBL in the contact guidance of neuronal migration, interneuronal recognition, formation of bridges between migrating neurons and radial astrocytes fibers, myelination and tightening of the ependymal cell barrier, during the ontogenic development of the brain. Based on these evidences, it is conceivable to hypothesize that polymorphisms in the MBL2 gene could suppress these functions, increasing the susceptibility of the brain tissue to various pathogenic insults, as infections and hemorrhages. This risk in our patients seems to be independent from infections, as was shown in the logistic regression. We did not find an association between genotype OO and PVL at multivariate analysis. However, considering the lack of brain MRI imaging in the majority of patients and being US insensitive in diagnosing the disease, this result could be unreliable.

This study was limited by a number of factors. First, although the acceptable statistical power, we have explored a rather large difference between the proportion of the genetic trait in cases and in controls. However, at our surprise, the difference we found was higher than expected, showing that further studies are needed to validate, better characterize, and define the medical relevance of these SNPs. Second, all patients were enrolled at a single institution, and bias may have been introduced by patient selection or institutional standards of care. However, to collect these types of data is very difficult. Finally, we lack a "normal" control population. In our study, this population should be constituted by at term infants, and the initial hypothesis of the study stated that mechanisms we have discussed may occur in conditions of severe prematurity at birth.

In conclusion, homozygosity for the allele O (SNPs of the exon-1) of the $M B L 2$ gene is associated with an increased risk of adverse neurological outcome at 24 mo in preterm infants, predicting neurological risks. These biallelic carriers of MBL coding variants are more exposed to the risk of brain hemorrhage during the hospitalization, apparently regardless of infections. Brain MRI evaluation of preterm neonates is important to better estimate the relationship between risk factors, MBL genotypes included, and PVL.

\section{METHODS}

In this longitudinal prospective study, 136 preterm infants of Caucasian ethnicity, with GA $\leq 32 \mathrm{wk}$, were consecutively admitted in our neonatal intensive care unit in 5 y (2005-2009) and observed from the admission to discharge. During the hospitalization, the following clinical and genetical data were recorded: gender, Apgar score at $5 \mathrm{~min}$, birth weight, GA, mode of delivery, days of mechanical ventilation with orotracheal tube (MV) and/or nasal continuous positive airway pressure presence of confirmed NS (with positive blood culture), necrotizing enterocolitis, IVH or PVL, and neonatal seizures. Infants discharged from the neonatal intensive care unit entered a 2 y follow-up program: 53 of them did not continue with the observation (Figure 1).

Genomic DNA was extracted from blood samples, using the QIAmp DNA Blood kit (Qiagen, Hilden, Germany). Genotyping of the MBL gene exon- 1 (mutant codons 52, 54, and 57) and promoter (position -221) was performed by polymerase chain reaction and restriction fragment length polymorphism. For exon-1, the wild-type allele was designated as A, whereas $\mathrm{B}, \mathrm{C}$, and $\mathrm{D}$ were, respectively, mutants in codon 54, 57, and 52. For the promoter, the wild-type allele was designated as Y (position -221), whereas X was the mutant. The three MBL-2 SNPs affecting the coding sequence $\mathrm{B}, \mathrm{C}$, and $\mathrm{D}$ were collectively referred as O. The Laboratory of Immunology (Anna Meyer Children's Hospital, Florence, Italy), in which the genotyping analyses were performed, joins the Leonardo's Program Quali VEQ (external 
quality control), (https://www.abanalitica.it/?link=dettaglio\&ID=3). All the genetic screening have been performed in duplicate and, when the genotype obtained was particularly rare, it was further analyzed. In every analysis, samples with known genotypes were included as positive controls.

During the follow-up program for $2 \mathrm{y}$, children were evaluated by clinical exams, brain US imaging at 3 and/or 6 mo of age, auditory brainstem response, and fundus examination. Cerebral hemorrhage was classified by a sonographic grading system, into four grades, according to Papile and Burstein (29). Serial electroencephalograms were performed when seizures were suspected.

Each child was examined in the outpatient clinic, and the assessment consisted of:

\section{Neurological Examination}

A clinical examination, including neurologic assessment, aimed to detect the presence of a neurological disability. It was defined as "severe neurological impaired" if the child had severe neuromotor delay or cerebral palsy. Cerebral palsy was diagnosed and classified according to the description of function for each limb in those with abnormal neurologic examination (30).

\section{Vision and Hearing Exams}

Visual function and retinal diseases were assessed by repeated examinations of the fundus and functional tests (electroretinogram and visual evoked potentials when necessary). Normal vision was defined as the "absence of any detectable pathology of the visual system"; mild abnormal vision as "the presence of a mild impairment which allowed useful vision", and severe visual impairment as "a child functionally blind or perceives light only". Hearing function was explored by auditory brainstem evoked potentials (ABR). Auditory global function was defined as normal in "absence of any detectable pathology", as mild if requiring hearing aids, or as severe if functionally deaf (uncorrected even with aids).

\section{Evaluation of Child Development}

Development was assessed with the use of Bayley Scales of Infant Development second Edition considering CA (31). Development was considered impaired if the scores in either the MDI or the PDI were equal or less than 70 .

The presence of a severe neurological impairment or an MDI and/ or PDI score $\leq 70$, at $24 \mathrm{mo}$, associated or not with the presence of a sensorial impairment was defined as "adverse neurological outcome" and was considered the main outcome of the study. An isolated defect of vision, hearing, or both, in the absence of abnormal neurological examination, or of MDI/PDI score $\leq 70$ was not defined as adverse neurological outcomes, because we could not exclude the influence of other different causes of sensory impairment (drug side effects, viral infections, retinopathy of prematurity).

The secondary outcome was cerebral hemorrhage diagnosed with US imaging.

In the end, 75 infants were observed, genotyped for $M B L 2$ gene, and followed up to $24 \mathrm{mo}$ of CA for neurological outcome measures.

The approval was obtained by the Ethics Committee of the Bambino Gesù Children's Hospital. The parents signed an informed consent at admission of the newborn in the neonatal intensive care unit.

\section{Statistical Analysis}

Continuous variables were described with mean \pm SD. Inferences about categorical data were analyzed with $\chi^{2}$ or Fisher exact test, as appropriate. To adjust for potential confounding variables, logistic regression analysis was performed, with IVH as dependent variable, entering in the model covariates with a $P$ value of $<0.05$ at univariate analysis. STATA software was used (version 10.0) for analysis and a $P$ value $<0.05$ was considered statistically significant. A number of 75 patients (with a case: control ratio of 1:2, i.e., 25 cases and 50 controls) was calculated as necessary to show a significant $(\alpha=0.05,1-\beta=0.8)$ difference of 0.30 between the estimated control group proportion of 0.67 , in agreement with the published prevalence of A/A, and the proportion of cases (20).

\section{STATEMENT OF FINANCIAL SUPPORT}

No funding was received to support this research.
Discloure: All the authors declare that they have participated in the concept and design and have approved the manuscript as submitted. All the authors have no conflict of interest in connection with this paper.

\section{REFERENCES}

1. Nour NM. Premature delivery and the millennium development goal. Rev Obstet Gynecol 2012;5:100-5.

2. Stoll BJ, Hansen NI, Bell EF, et al.; Eunice Kennedy Shriver National Institute of Child Health and Human Development Neonatal Research Network. Neonatal outcomes of extremely preterm infants from the NICHD Neonatal Research Network. Pediatrics 2010;126:443-56.

3. Volpe JJ. Brain injury in premature infants: a complex amalgam of destructive and developmental disturbances. Lancet Neurol 2009;8:110-24.

4. Garred P, Larsen F, Seyfarth J, Fujita R, Madsen HO. Mannose-binding lectin and its genetic variants. Genes Immun 2006;7:85-94.

5. Sorensen GL, Petersen I, Thiel S, et al. Genetic influences on mannanbinding lectin $(\mathrm{MBL})$ and mannan-binding lectin associated serine protease-2 (MASP-2) activity. Genet Epidemiol 2007;31:31-41.

6. Madsen HO, Garred P, Thiel S, et al. Interplay between promoter and structural gene variants control basal serum level of mannan-binding protein. J Immunol 1995;155:3013-20.

7. Fujita T, Matsushita M, Endo Y. The lectin-complement pathway-its role in innate immunity and evolution. Immunol Rev 2004;198:185-202.

8. Maródi L. Neonatal innate immunity to infectious agents. Infect Immun 2006;74:1999-2006.

9. Ip WK, Takahashi K, Ezekowitz RA, Stuart LM. Mannose-binding lectin and innate immunity. Immunol Rev 2009;230:9-21.

10. Wang ZY, Sun ZR, Zhang LM. The relationship between serum mannosebinding lectin levels and acute ischemic stroke risk. Neurochem Res 2014;39:248-53.

11. Osthoff M, Katan M, Fluri F, et al. Mannose-binding lectin deficiency is associated with smaller infarction size and favorable outcome in ischemic stroke patients. PLoS One 2011;6:e21338.

12. Cervera A, Planas AM, Justicia C, et al. Genetically-defined deficiency of mannose-binding lectin is associated with protection after experimental stroke in mice and outcome in human stroke. PLoS One 2010;5:e8433.

13. del Zoppo G, Ginis I, Hallenbeck JM, Iadecola C, Wang X, Feuerstein GZ. Inflammation and stroke: putative role for cytokines, adhesion molecules and iNOS in brain response to ischemia. Brain Pathol 2000;10:95-112.

14. Zhang M, Takahashi K, Alicot EM, et al. Activation of the lectin pathway by natural IgM in a model of ischemia/reperfusion injury. J Immunol 2006;177:4727-34.

15. Lau YL, Chan SY, Turner MW, Fong J, Karlberg J. Mannose-binding protein in preterm infants: developmental profile and clinical significance. Clin Exp Immunol 1995;102:649-54.

16. Thiel S, Bjerke T, Hansen D, Poulsen LK, Schiøtz PO, Jensenius JC. Ontogeny of human mannan-binding protein, a lectin of the innate immune system. Pediatr Allergy Immunol 1995;6:20-3.

17. Hilgendorff A, Schmidt R, Bohnert A, Merz C, Bein G, Gortner L. Host defence lectins in preterm neonates. Acta Paediatr 2005;94:794-9.

18. Frakking FN, Brouwer N, Zweers D, et al. High prevalence of mannosebinding lectin (MBL) deficiency in premature neonates. Clin Exp Immunol 2006;145:5-12.

19. Catsburg A, van der Zwet WC, Morré SA, Ouburg S, VandenbrouckeGrauls CM, Savelkoul PH. Analysis of multiple single nucleotide polymorphisms (SNP) on DNA traces from plasma and dried blood samples. J Immunol Methods 2007;321:135-41.

20. Yager PH, You Z, Qin T, et al. Mannose binding lectin gene deficiency increases susceptibility to traumatic brain injury in mice. J Cereb Blood Flow Metab 2008: 1-10.

21. Ducruet AF, Sosunov SA, Zacharia BE, et al. The neuroprotective effect of genetic mannose-binding lectin deficiency is not sustained in the subacute phase of stroke. Transl Stroke Res 2011;2:588-99.

22. Heyer EJ, Kellner CP, Malone HR, et al. Complement polymorphisms and cognitive dysfunction after carotid endarterectomy. J Neurosurg 2013;119:648-54.

23. Annells MF, Hart PH, Mullighan CG, et al. Interleukins-1, -4, -6, -10, tumor necrosis factor, transforming growth factor-beta, FAS, and man- 
nose-binding protein $\mathrm{C}$ gene polymorphisms in Australian women: Risk of preterm birth. Am J Obstet Gynecol 2004;191:2056-67.

24. Bodamer OA, Mitterer G, Maurer W, Pollak A, Mueller MW, Schmidt WM. Evidence for an association between mannose-binding lectin 2 (MBL2) gene polymorphisms and pre-term birth. Genet Med 2006;8: $518-24$.

25. van Vliet EO, de Kieviet JF, Oosterlaan J, van Elburg RM. Perinatal infections and neurodevelopmental outcome in very preterm and very low-birth-weight infants: a meta-analysis. JAMA Pediatr 2013;167: 662-8.

26. Zanetta JP. Mannose binding lectin in cerebrum development. Progress in Molecular and Subcellular Biology 2003;32:75-96. Kostovic I (ed). Berlin Heidelberg: Springer-Verlag.
27. Reeber A, Vincendon G, Zanetta JP. Transient concanavalin A-binding glycoproteins of the parallel fibres of the developing rat cerebellum: evidence for the destruction of their glycans. J Neurochem 1980;35:1273-7.

28. Reeber A, Vincendon G, Zanetta JP. Isolation and immunohistochemical localization of a "Purkinje cell specific glycoprotein subunit from rat cerebellum. Brain Res 1981;229:53-65.

29. Papile LA, Burstein J, Burstein R, Koffler H. Incidence and evolution of subependymal and intraventricular hemorrhage: a study of infants with birth weights less than 1,500 gm. J Pediatr 1978;92:529-34.

30. Evans P, Alberman E, Johnson A, Mutch L. Standardisation of recording and reporting cerebral palsy. Dev Med Child Neurol 1987;29:272.

31. Bayley N. Bayley Scales of Infant Development. Second Edition Manual. San Antonio, TX: The Psychological Corporation, 1993. 